\title{
STABILIZATION OF ELASTIC PLATES \\ WITH VARIABLE COEFFICIENTS AND DYNAMICAL BOUNDARY CONTROL
}

\author{
$\mathrm{BY}$ \\ YUXIA GUO (Department of Mathematics, Tsinghua University, Beijing 100084, China), \\ SHUGEN CHAI (Institute of Systems Science, Academy of Mathematics and Systems Sciences, \\ Chinese Academy of Sciences, Beijing 100080, P.R. China and Department of Mathematics, \\ Shanxi University, Taiyuan 03006, China), \\ AND \\ PENGFEI YAO (Institute of Systems Science, Academy of Mathematics and Systems Sciences, \\ Chinese Academy of Sciences, Beijing 100080, P.R. China)
}

\begin{abstract}
The aim of this paper is to investigate the stabilization of a hybrid system composed of a plate equation with variable coefficients and two ordinary differential equations under some suitable feedbacks. A rational energy decay rate is established by the multiplier method and the Riemannian geometry method, and the uniform energy decay rate for a simplified system is obtained.
\end{abstract}

1. Introduction. We consider the stabilization problem of a hybrid system with variable coefficients where, for convenience, our problems start out on a Riemannian manifold $M$ of dimension 2 with a metric $g=\langle\cdot, \cdot\rangle$. For the classical case where $M=R^{2}$ and $g$ is the dot product, the problem mentioned above has been well studied by Rao [1] and others. Here we use the Riemannian geometry method to obtain the stabilization results for the elastic plate with variable coefficients and dynamical boundary control. This method is first introduced into the boundary control problem by Yao [2] for the wave equation.

Our paper is organized as follows. In Sec. 2, we introduce some notation with which we are working. In Sec. 3, we establish the rational energy decay rate for the smooth solution to the system. In Sec. 4, we consider a simplified plate model, and obtain the uniform energy decay rate of the system.

Received September 6, 2000.

2000 Mathematics Subject Classification. Primary 35B40, 35M10, 93C15, 93C20, 93D15.

Key words and phrases. hybrid system, boundary feedback, rational energy decay rate, Riemannian geometry method.

This work is supported by the CAS K. C. Wang Post-doctoral Research Award Fund and the National Science Foundation of China and the National Key Project of China.

E-mail address: yguo@math.tsinghua.edu.cn 
2. Some notation. We introduce some notation in the Riemannian manifold in preparation for our system of the elastic plate with dynamical boundary control. It should be mentioned that all definitions and notation in this section are standard and classical in the literature.

Let $(M, g)$ be a Riemannian manifold with Riemannian metric $g=\langle\cdot, \cdot\rangle$. For each $x \in M, M_{x}$ is the tangential space of $M$ at $x$. We use $\chi(M)$ for the set of all vector fields on $M$. Denote the set of all $k$-order tensor fields and all $k$-forms on $M$ by $T^{k}(M)$ and $\Lambda^{k}(M)$ respectively, where $k$ is a nonnegative integer.

It is well known that, for each $x \in M$, the $k$-order tensor space $T_{x}^{k}$ on $M_{x}$ is an inner product space, and its inner product $\langle\cdot, \cdot\rangle$ is defined in the following way. Let $e_{1}, e_{2}$ be an orthonormal basis of $M_{x}$, and for any $\alpha, \beta \in T_{x}^{k}, x \in M$, define

$$
\langle\alpha, \beta\rangle_{T_{x}^{k}}=\sum_{i_{1}, i_{2}, \ldots, i_{k}=1}^{2} \alpha\left(e_{i_{1}}, \ldots, e_{i_{k}}\right) \beta\left(e_{i_{1}}, \ldots, e_{i_{k}}\right) \text { at } x .
$$

Let $\Omega$ be a bounded region of $M$ with a regular boundary $\Gamma$. Then $T^{k}(\Omega)$ is an inner product space with inner product $(\cdot, \cdot)$ in the following sense:

$$
\left(T_{1}, T_{2}\right)_{T^{k}(\Omega)}=\int_{\Omega}\left\langle T_{1}, T_{2}\right\rangle_{T_{x}^{k}} d x, \quad T_{1}, T_{2} \in T^{k}(\Omega),
$$

where $d x$ is the volume element of $M$ in its Riemannian metric $g$.

The completion of $T^{k}(\Omega)$ in the inner product $(2.2)$ is denoted by $L^{2}\left(\Omega, T^{k}\right)$. In particular, $L^{2}(\Omega, \Lambda)=L^{2}(\Omega, T)$. The completion of $C^{\infty}(\Omega)$ in the following inner product is defined by $L^{2}(\Omega)$ :

$$
(f, h)_{L^{2}(\Omega)}=\int_{\Omega} f(x) h(x) d x, \quad f, h \in C^{\infty}(\Omega) .
$$

Let $D$ be the Levi-Civita connection on $M$ in the Riemannian metric $g$. For $U \in$ $\chi(M), D U$ is the covariant differential of $U$, which is a second-order covariant tensor field in the following sense:

$$
D U(X, Y)=D_{Y} U(X)=\left\langle D_{Y} U, X\right\rangle, \quad \forall X, Y \in M_{x}, \quad x \in M .
$$

For any $T \in T^{2}(M)$, the trace of $T$ at $x$ is defined by

$$
\operatorname{tr} T=\sum_{i=1}^{2} T\left(e_{i}, e_{i}\right)
$$

where $e_{1}, e_{2}$ is an orthonormal basis of $M_{x}$. It is obvious that $\operatorname{tr} T \in C^{\infty}(M)$ if $T \in$ $T^{2}(M)$. The exterior derivative $d: \Lambda^{k}(M) \rightarrow \Lambda^{k+1}(M)$ satisfies $d^{2}=0$. There is a first-order differential operator $\delta: \Lambda^{k+1}(M) \rightarrow \Lambda^{k}(M)$, which is the formal adjoint of $d$ and characterized by

$$
\left.(d \alpha, \beta)_{L^{2}\left(\Omega, \Lambda^{k+1}\right.}\right)=(\alpha, \delta \beta)_{L^{2}\left(\Omega, \Lambda^{k}\right)},
$$

for $\alpha \in \Lambda^{k}(\Omega)$ and $\beta \in \Lambda^{k+1}(\Omega)$ with compact support.

The Sobolev space $H^{k}(\Omega)$ is the completion of $C^{\infty}(\Omega)$ with respect to the norm $\|f\|_{H^{k}(\Omega)}^{2}=\sum_{i=1}^{k}\left\|D^{i} f\right\|_{L^{2}\left(\Omega, T^{k}\right)}^{2}+\|f\|_{L^{2}(\Omega)}^{2}$, for $f \in C^{\infty}(\Omega)$, where $D^{i} f$ is the $i$ th covariant differential of $f$ in the metric $g$, and $\|\cdot\|_{L^{2}\left(\Omega, T^{k}\right)},\|\cdot\|_{L^{2}(\Omega)}$ are the induced 
norms in the inner products (2.2), (2.3), respectively. For details on the Sobolev spaces on the Riemannian manifolds, we refer to Hebey [3] or Taylor [4].

The following Green formulae are due to Taylor [4, Chapter 2, §10]:

$$
(d \alpha, \beta)_{L^{2}\left(\Omega, \Lambda^{k+1}\right)}=(\alpha, \delta \beta)_{L^{2}\left(\Omega, \Lambda^{k}\right)}+\int_{\Gamma}\langle\nu \wedge \alpha, \beta\rangle_{T_{x}^{k}+1} d \Gamma
$$

for $\alpha \in \Lambda^{k}(\bar{\Omega})$ and $\beta \in \Lambda^{k+1}(\bar{\Omega})$ and

$$
(\delta \alpha, \beta)_{L^{2}\left(\Omega, \Lambda^{k}\right)}=(\alpha, d \beta)_{L^{2}\left(\Omega, \Lambda^{k+1}\right)}-\int_{\Gamma}\left\langle l_{\nu} \wedge \alpha, \beta\right\rangle_{T_{x}^{k}} d \Gamma
$$

for $\alpha \in \Lambda^{k+1}(\bar{\Omega})$ and $\beta \in \Lambda^{k}(\bar{\Omega})$, where $d \Gamma$ is the line element of $\Gamma$ and $\nu$ is the unit normal of $\Gamma$ pointing towards the exterior of $\Gamma$. For $\alpha \in \Lambda^{k+1}(\bar{\Omega})$ and the unit normal $\nu, l_{\nu} \alpha \in T^{k}(\bar{\Omega})$ is defined by $l_{\nu} \alpha\left(X_{1}, \ldots, X_{k}\right)=\alpha\left(\nu, X_{1}, \ldots, X_{k}\right), \forall X_{1}, \ldots, X_{k} \in \chi(\bar{\Omega})$ and $\wedge$ is the exterior product of differential forms.

In the case of dimension 2, the Ricci tensor is a second-order covariant tensor field, given by

$$
\operatorname{Ricci}(X, Y)(x)=\sum_{i=1}^{2} R\left(e_{i}, X, e_{i}, Y\right), \quad \forall X, Y \in M_{x}, \quad x \in M,
$$

where $e_{1}, e_{2}$ is an orthonormal basis of $M_{x}$ and $R$ is the curvature tensor of the Levi-Civita connection (for details, see $\mathrm{Wu}[5]$ ). It is easy to check from (2.8) that

$$
\operatorname{Ricci}(X, Y)=k(x)\langle X, Y\rangle \quad \forall X, Y \in M_{x}, \quad x \in M,
$$

where $k(x)$ is the Gaussian curvature function on $M$. We denote by $\Delta: C^{2}\left(R^{2}\right) \rightarrow C\left(R^{2}\right)$ the Laplace operator in the Riemannian metric $g$. Then we have

$$
\Delta h=\frac{1}{\sqrt{G(x)}} \sum_{i, j=1}^{2} \frac{\partial}{\partial x_{i}}\left(\sqrt{G(x)} g^{i j}(x) \frac{\partial h}{\partial x_{j}}\right), \quad \forall h \in C^{2}\left(R^{2}\right),
$$

where $g_{i j}=g\left(\frac{\partial}{\partial x^{i}}, \frac{\partial}{\partial x^{j}}\right), G(x)=\operatorname{det}\left(g_{i j}\right)$, and $g_{i k} g^{k j}=\delta_{i}^{j}, \quad x=\left(x_{1}, x_{2}\right)$ is the classical coordinate system.

It follows from Yao [2, Lemma 2.1] that

$$
\Delta h=\sum_{i, j=1}^{2} g^{i j} D^{2} h\left(\frac{\partial}{\partial x^{i}}, \frac{\partial}{\partial x^{j}}\right), \quad \forall h \in C^{2}\left(R^{2}\right) .
$$

We will use many times the following divergence formulae:

$$
\int_{\Omega} \operatorname{div} X d x=\int_{\Gamma}\langle X, \nu\rangle d \Gamma
$$

where $\operatorname{div} X$ is the divergence of the vector field $X$ in the Riemannian metric $g$, and $\nu$ is the normal of $\Gamma$ pointing towards the exterior of $\Gamma$. 
3. Rational energy decay rate. We keep all the notation as in Sec. 2 . Let $\Omega$ denote a bounded domain in the Riemannian manifold $(M, g)$ with smooth boundary $\Gamma$ consisting of two disjoint parts $\Gamma_{0} \cup \Gamma_{1}=\Gamma$. Here we will consider a curvy plate with the dynamic boundary whose middle surface, $\Omega$, is part of a surface $M$ and where the extension effects along the tangential direction are neglected. We assume that the material undergoing change obeys Hooke's law. Then the potential energy is to be defined by

$$
P_{\Omega}=\int_{\Omega}\left[(1-\mu)\left|D^{2} y\right|^{2}+\mu\left(\operatorname{tr} D^{2} y\right)^{2}\right] d x
$$

where $y$ is the displacement of the plate along the normal and $0<\mu<\frac{1}{2}$ is the Poisson coefficient. The energy produced by the dynamic boundary is to be defined by

$$
P_{\Gamma}=J \int_{\Gamma_{1}}\left|\partial_{\nu} y^{\prime}\right|^{2} d \Gamma+\rho \int_{\Gamma_{1}}\left|y^{\prime}\right|^{2} d \Gamma,
$$

where $\rho>0$ is the linear boundary density and $J>0$ is the bending moment of inertia of the boundary.

If there is no external force, then the equations of motion for $y$ are obtained by setting to zero the first variation of the Lagrangian:

$$
\int_{0}^{T}\left[\left|y^{\prime}\right|^{2}-P_{\Omega}(y)-P_{\Gamma_{1}}(y)\right] d t
$$

(the "Principle of the Virtual Work"). Then the variation of (3.3) is taken with respect to kinematically admissible displacements.

We obtain, as the result of calculation by the variation of (3.3), the following system:

$$
\begin{array}{ll}
y^{\prime \prime}+\Delta^{2} y-(1-\mu) \delta(k d y)=0 & \text { in } \Omega \times[0, \infty), \\
y=\partial_{\nu} y=0 & \text { on } \Gamma_{0} \times[0, \infty), \\
J \partial_{\nu} y^{\prime \prime}+\Delta y+(1-\mu) B_{1} y=0 & \text { on } \Gamma_{1} \times[0, \infty), \\
\rho y^{\prime \prime}-\partial_{\nu} \Delta y-(1-\mu) B_{2} y=0 & \text { on } \Gamma_{1} \times[0, \infty), \\
y(0)=y_{0}, \quad y^{\prime}(0)=y_{1} & \text { on } \Omega,
\end{array}
$$

where $\nu$ is the unit normal along $\Gamma$ pointing towards the exterior of $\Gamma$ and $\Delta: C^{2}(M) \rightarrow$ $C(M)$ is the Laplace operator in the Riemannian metric $g$. In the above equations, $k$ is the Gaussian curvature function on $M ; d$ is the exterior derivative; $\delta$ is the formal adjoint of $d ; B_{1}, B_{2}$ are the boundary operators defined by

$$
B_{1} y=-D^{2} y(\tau, \tau)
$$

and

$$
B_{2} y=\frac{\partial}{\partial \tau}\left(D^{2} y(\tau, \nu)\right)+k \partial_{\nu} y
$$

respectively, where $D^{2} y$ is the Hessian of $y$, which is a second-order tensor, $\tau$ is the tangential along curve $\Gamma$, and $\partial_{\nu} y=\frac{\partial y}{\partial \nu}=\langle\nu, D y\rangle$. In this section, we will consider the 
control problem:

$$
\begin{array}{ll}
y^{\prime \prime}+\Delta^{2} y-(1-\mu) \delta(k d y)=0 & \text { in } \Omega \times[0, \infty), \\
y=\partial_{\nu} y=0 & \text { on } \Gamma_{0} \times[0, \infty), \\
J \partial_{\nu} y^{\prime \prime}+\Delta y+(1-\mu) B_{1} y=m & \text { on } \Gamma_{1} \times[0, \infty), \\
\rho y^{\prime \prime}-\partial_{\nu} \Delta y-(1-\mu) B_{2} y=f & \text { on } \Gamma_{1} \times[0, \infty), \\
y(0)=y_{0}, \quad y^{\prime}(0)=y_{1} & \text { on } \Omega,
\end{array}
$$

where $m, f$ are the feedbacks to be defined by

$$
m=-\partial_{\nu} y^{\prime}, \quad f=-y^{\prime} .
$$

Also, we will establish the rational energy decay rate for the smooth solution of system (3.6) and (3.7).

REMARK 3.1. The term $(1-\mu) \delta(k d y)$ in the system (3.6) comes from the curvedness of the metric. For the flat case where $M=R^{2}$ and $k=0$, system (3.6) is the same as in Rao [1].

Well posedness and regularity. In the following, we will discuss briefly the well posedness and the smoothness of solutions to the system (3.6) and (3.7). The idea is the same as in Rao [1] since the variable coefficients do not influence the regularity of the problem.

Let

$$
A y=\Delta^{2} y-(1-\mu) \delta(k d y) .
$$

By Lemma 3.1 below, the equation in (3.6) then becomes

$$
y^{\prime \prime}+A y=0 \text {. }
$$

By a similar argument in the sense of a semigroup of contractions in [1], we can obtain the existence and uniqueness of a solution to the system (3.6) and (3.7).

Let $S=\left\{u=(y, z, \xi, \eta) \in W \times H_{\Gamma_{0}}^{2}(\Omega) \times L^{2}\left(\Gamma_{1}\right) \times L^{2}\left(\Gamma_{1}\right)\right\}$ such that $\xi=\left.\partial_{\nu} z\right|_{\Gamma_{1}}$ and $\eta=\left.z\right|_{\Gamma_{1}}$, where $W$ is defined by

$$
W=\left\{\begin{array}{l}
y \in H_{\Gamma_{0}}^{2}(\Omega), \Delta^{2} y \in L^{2}(\Omega), \\
\Delta y+(1-\mu) B_{1} y=v_{1} \in L^{2}\left(\Gamma_{1}\right), \\
\partial_{\nu} \Delta y+(1-\mu) B_{2} y=v_{2} \in L^{2}\left(\Gamma_{1}\right) .
\end{array}\right.
$$

If the initial data $u_{0} \in S$, then system (3.6) has a solution $y$ satisfying

$$
\begin{aligned}
& y(t) \in C^{0}\left(R^{+} ; H^{\frac{5}{2}}\right) \cap C^{1}\left(R^{+} ; H_{\Gamma_{0}}^{2}(\Omega)\right) \cap C^{2}\left(R^{+} ; L^{2}(\Omega)\right) ; \\
& \left.y\right|_{\Gamma_{1}} \in C^{2}\left(R^{+} ; L^{2}\left(\Gamma_{1}\right)\right) ;\left.\quad \partial_{\nu} y\right|_{\Gamma_{1}} \in C^{2}\left(R^{+} ; L^{2}\left(\Gamma_{1}\right)\right) .
\end{aligned}
$$

By the elliptic theory, we can obtain the regularity of the solutions to the system (3.6) and (3.7). In fact, if initial data $y(0)=y_{0} \in H_{\Gamma_{0}}^{4 k+2}(\Omega), y_{t}(0)=y_{1} \in H_{\Gamma_{0}}^{4 k+2}(\Omega)$, for $k \geq 1$, then we have $\left(y_{0}, y_{1},\left.\partial_{\nu} y_{1}\right|_{\Gamma_{1}},\left.y_{1}\right|_{\Gamma_{1}}\right) \in S$. Therefore, system (3.6) has a solution $y(t)$ satisfying

$$
\left(y(0), y_{t}(0),\left.\partial_{\nu} y_{t}(0)\right|_{\Gamma_{1}},\left.y_{t}^{(0)}\right|_{\Gamma_{1}}\right)=\left(y_{0}, y_{1},\left.\partial_{\nu} y_{1}\right|_{\Gamma_{1}},\left.y_{1}\right|_{\Gamma_{1}}\right)
$$


On the other hand, we have

$$
\begin{aligned}
& y_{t t}(0)=-A y(0) \in H_{\Gamma_{0}}^{4 k-2}(\Omega) \\
& y_{t}(0)=y_{1} \in H_{\Gamma_{0}}^{4 k+2}(\Omega) \subset H_{\Gamma_{0}}^{2}(\Omega) .
\end{aligned}
$$

Set $\phi=y_{t}$. Then $\phi$ satisfies

$$
\left\{\begin{array}{l}
\phi_{t t}+A \phi=0, \\
\left.\phi\right|_{\Gamma_{0}}=\left.\partial_{\nu} \phi\right|_{\Gamma_{0}}=0, \\
J \partial_{\nu} \phi^{\prime \prime}+\Delta \phi+(1-\mu) B_{1} \phi=m_{1}, \\
\rho \phi^{\prime \prime}-\partial_{\nu} \Delta \phi-(1-\mu) B_{2} \phi=f_{1},
\end{array}\right.
$$

with initial data $\left(y_{t}(0), y_{t t}(0),\left.\partial_{\nu} y_{t t}(0)\right|_{\Gamma_{1}},\left.y_{t t}(0)\right|_{\Gamma_{1}}\right) \in S$.

From the system (3.6), we obtain that $y_{t t}$ is a strong solution of the system with initial data $\left(y_{t}(0), y_{t t}(0),\left.\partial_{\nu} y_{t t}(0)\right|_{\Gamma_{1}},\left.y_{t t}(0)\right|_{\Gamma_{1}}\right) \in S$, and therefore we have

$$
y_{t t} \in C^{2}\left(R^{+} ; L^{2}(\Omega)\right),\left.\quad y_{t t}\right|_{\Gamma_{1}} \in C^{2}\left(R^{+} ; L^{2}\left(\Gamma_{1}\right)\right),\left.\quad \partial_{\nu} y_{t t}\right|_{\Gamma_{1}} \in C^{2}\left(R^{+} ; L^{2}\left(\Gamma_{1}\right)\right),
$$

and

$$
\left\{\begin{array}{l}
\left(y_{t t}\right)_{t t}+A y_{t t}=0 \\
\Delta y_{t t}+(1-\mu) B_{1} y_{t t}=-\partial_{\nu}\left(y_{t t}\right)_{t}-J \partial_{\nu}\left(y_{t t}\right)_{t t}, \\
-\partial_{\nu} \Delta y_{t t}-(1-\mu) B_{2} y_{t t}=-\left(y_{t t}\right)_{t}-\rho\left(y_{t t}\right)_{t t}
\end{array}\right.
$$

By system (3.6) and (3.8), we have

$$
\left\{\begin{array}{l}
A^{2} y=\left(y_{t t}\right)_{t t} \in L^{2}(\Omega) \\
\Delta y+(1-\mu) B_{1} y=-\partial_{\nu} y_{t}-J \partial_{\nu} y_{t t} \in H^{2}\left(\Gamma_{1}\right), \\
-\partial_{\nu} \Delta y-(1-\mu) B_{2} y=-y_{t}-\rho y_{t t} \in H^{2}\left(\Gamma_{1}\right) .
\end{array}\right.
$$

It is easy to check that $A$ is an elliptic operator (see Taylor [4]). Thus from elliptic theory, we obtain $y \in H^{\frac{9}{2}}(\Omega)$. If the initial data have more regularity, then we obtain more regularity of solutions by repeating the above steps.

The following formula is key to our problems, which is something like the classical Green's formula presenting the relationship between the interior and the boundary.

LEMmA 3.1. Let $y, u \in H^{4}(\Omega)$ be given such that all the terms in the following formulae are well defined, where $\Gamma$ is a closed curve. Then we have

$$
\begin{aligned}
\int_{\Omega} & {\left[\Delta^{2} y-(1-\mu) \delta(k d y)\right] u d x=\int_{\Omega} a(y, u) d x } \\
- & \int_{\Gamma}\left[\Delta y+(1-\mu) B_{1} y\right] \frac{\partial u}{\partial \nu} d \Gamma+\int_{\Gamma}\left[\frac{\partial(\Delta y)}{\partial \nu}+(1-\mu) B_{2} y\right] u d \Gamma,
\end{aligned}
$$

where

$$
a(y, u)=(1-\mu)\left\langle D^{2} y, D^{2} u\right\rangle_{T_{x}^{2}}+\mu\left(\operatorname{tr} D^{2} y \operatorname{tr} D^{2} u\right) .
$$

Proof. Since $y$ is a function, we have $\delta y=0$, and

$$
\delta \Delta_{H} d y=\delta d \delta d y=\Delta_{H}^{2} y
$$


where $\Delta_{H}$ is the Hodge-Laplacian on forms, and $\Delta_{H}=-\Delta$ if it is applied to functions and $\Delta_{H} d y=-d \Delta y$.

Since $d y=D y$, it follows from Yao [6, Theorem 2.2], and formulae (2.6), (3.11) that

$$
\begin{aligned}
\int_{\Omega} & \left\langle D^{2} y, D^{2} u\right\rangle_{T_{x}^{2}} d x \\
= & (D d y, D d u)_{L^{2}\left(\Omega, T^{2}\right)}=\int_{\Omega}\langle D d y, D d u\rangle d x \\
= & \int_{\Omega}\left[\left\langle\Delta_{H} d y-k d y, d u\right\rangle d x+\int_{\Gamma}\left\langle D_{\nu} d y, d u\right\rangle d \Gamma,\right. \\
= & \int_{\Omega}\left(\Delta_{H}^{2} y-\delta(k d y)\right) u d x \\
& +\int_{\Gamma} u\left[\left\langle\nu, \Delta_{H} d y\right\rangle-k \frac{\partial y}{\partial \nu}\right] d \Gamma+\int_{\Gamma} D^{2} y(\nu, d u) d \Gamma \\
= & \int_{\Omega}\left[\left(\Delta^{2} y-\delta(k d y)\right) u\right] d x \\
& +\int_{\Gamma}\left[D^{2} y(\nu, \nu) \frac{\partial u}{\partial \nu}+D^{2} y(\nu, \tau) \frac{\partial u}{\partial \tau}\right] d \Gamma-\int_{\Gamma} u\left[\frac{\partial \Delta y}{\partial \nu}+k \frac{\partial y}{\partial \nu}\right] d \Gamma .
\end{aligned}
$$

Since $\operatorname{tr} D^{2} y=\Delta y$, by Green's formula, we have

$$
\int_{\Omega} \operatorname{tr} D^{2} y \operatorname{tr} D^{2} u d x=\int_{\Omega} \Delta^{2} y u d x+\int_{\Gamma}\left[\Delta y \frac{\partial u}{\partial \nu}-u \frac{\partial \Delta y}{\partial \nu}\right] d \Gamma .
$$

Since $\Gamma$ is a closed curve,

$$
\int_{\Gamma} D^{2} y(\nu, \tau) \frac{\partial u}{\partial \tau} d \Gamma=-\int_{\Gamma} u \frac{\partial}{\partial \tau}\left(D^{2} y(\nu, \tau)\right) d \Gamma
$$

Furthermore, we have

$$
\Delta y=D^{2} y(\nu, \nu)+D^{2} y(\tau, \tau) \quad \text { on } \Gamma .
$$

By (3.11)-(3.15) and (3.10), we get formulae (3.9).

Let $H$ be a vector field on the Riemannian manifold $(M, g)$ such that

$$
D H(X, X)=b(x)|X|^{2} \quad \forall X \in M_{x}, x \in \bar{\Omega},
$$

where $b(x)$ is a function on $\Omega$. We also assume that the vector $H$ satisfies

$$
b_{0}=\min _{x \in \Omega} b(x)>0
$$

and

$$
\langle H, \nu\rangle \leq 0, \quad \forall x \in \Gamma_{0} ; \quad\langle H, \nu\rangle>0 \quad \forall x \in \Gamma_{1} .
$$

We say that the vector field $H$ satisfies Assumption $\mathbf{A}$ if $H$ is such that relations (3.16)(3.18) hold. We say that the vector field $H$ satisfies Assumption $\mathbf{B}$ if $H$ is such that not only conditions (3.16)-(3.18) hold but also the following inequality is true:

$$
2 \theta_{1}\left(\theta_{2}+\theta_{3}\right)<1
$$


where

$$
\begin{aligned}
& \theta_{2}=\max _{x \in \Omega}|k||H(x)|, \\
& \theta_{3}=\max _{x \in \Omega}\left|D^{2} H(x)\right|,
\end{aligned}
$$

$k$ is the Gauss curvature function, and $\theta_{1}$ is the best constant such that the following inequality is true:

$$
\int_{\Omega}|D y|^{2} d x \leq \theta_{1}^{2} \int_{\Omega} a(y, y) d x \quad \forall y \in H_{\Gamma_{0}}^{2}(\Omega) .
$$

REMARK 3.2. The geometric condition (3.16) is used in Yao [7] for some observability inequalities of the Euler-Bernoulli equation with variable coefficients. For any Riemannian manifold, the existence of such a vector field on $\Omega \subset M$ has been proved by Yao [8]. Assumption $\mathbf{A}$ is enough to get the uniform stabilization of the simplified model; see Theorem 4.1 in Sec. 4. However, since we encounter difficulties when we try to use the traditional method of compactness-uniqueness to eliminate the lower term in the proof of Theorem 3.1 below, we make assumption (3.19) to overcome it. For the classical case where $H=x-x_{0}$ and $k=0$, Assumption $\mathbf{B}$ is true since $D^{2} H=0$, and therefore we have $\theta_{1}\left(\theta_{2}+\theta_{3}\right)=0$. One can also find some other nontrivial examples in Yao [8] to satisfy Assumption $\mathbf{B}$.

Furthermore, set

$$
T(G, F)=(1-\mu)\langle G, F\rangle+\mu \operatorname{tr} G \operatorname{tr} F,
$$

where $G, F$ are second-order tensors and

$$
L(y)=R(D y, \cdot, H, \cdot)+D^{2} H(D y, \cdot, \cdot)
$$

where "." denotes the position of the variable. It is easy to check that $a(y, y)=$ $T\left(D^{2} y, D^{2} y\right)$.

LEMma 3.2. Letting $H$ satisfy (3.16), we have

$$
\begin{gathered}
\int_{\Omega} a(y, H(y)) d x=\frac{1}{2} \int_{\Gamma} a(y, y)\langle H, \nu\rangle d \Gamma+\int_{\Omega} b a(y, y) d x \\
+\int_{\Omega} T\left(D^{2} y, L(y)\right) d x .
\end{gathered}
$$

Proof. Given $x \in \Omega$, let $E_{1}, E_{2}$ be a normal frame field normal at $x$. By the following identity (see Wu $[5, \S 2$, Lemma 4]),

$$
D^{2} T(\cdots, X, Y)=D^{2} T(\cdots, Y, X)+\left(R_{X Y} T\right)(\cdots),
$$

we have

$$
\begin{aligned}
D^{2}( & H(y))\left(E_{i}, E_{j}\right) \\
= & E_{j} E_{i}\left(D_{y}(H)\right)=E_{j}\left(D^{2} y\left(E_{i}, H\right)+D y\left(D_{E_{i}} H\right)\right) \\
= & D^{3} y\left(E_{i}, H, E_{j}\right)+D^{2} y\left(E_{i}, D_{E_{j}} H\right)+E_{j}\left\langle D_{y}, D_{E_{i}} H\right\rangle \\
= & D_{H}\left(D^{2} y\right)\left(E_{i}, E_{j}\right)+R\left(D y, E_{i}, H, E_{j}\right) \\
& +D^{2} y\left(E_{i}, D_{E_{j}} H\right)+E_{j}\left\langle D_{y}, D_{E_{i}} H\right\rangle \quad \text { at } x .
\end{aligned}
$$


Since $\left(D_{E_{i}} E_{j}\right)(x)=0$ for $1 \leq i, j \leq 2$,

$$
\begin{aligned}
E_{j}\left\langle D_{y}, D_{E_{i}} H\right\rangle & =D^{2} H\left(D y, E_{i}, E_{j}\right)+D H\left(D_{E_{j}} D y, E_{i}\right) \\
& =l_{D y} D^{2} H\left(E_{i}, E_{j}\right)+D^{2} y\left(D_{E_{i}} H, E_{j}\right) \text { at } x .
\end{aligned}
$$

Inserting (3.26) into (3.25) yields

$$
D^{2}(H(y))=D_{H}\left(D^{2} y\right)+D^{2} y(\cdot, D \cdot H)+D^{2} y(D \cdot H, \cdot)+L(y) .
$$

On the other hand, given $x \in \Omega$, let $E_{1}, E_{2}$ be a frame field normal at $x$. By direct computation, we have

$$
H\left(\operatorname{tr} D^{2} y\right)=\sum_{i=1}^{2} D_{H} D^{2} y\left(E_{i}, E_{i}\right)=\operatorname{tr}\left(D_{H} D^{2} y\right) \quad \text { at } x .
$$

Since $D^{2} y$ is a symmetric, second-order tensor field, we have by Yao [8, Prop. 2.1] and formula (3.27)

$$
\begin{aligned}
& \left\langle D^{2} y, D^{2}(H(y))\right\rangle_{T_{x}^{2}}=\frac{1}{2} H\left(\left|D^{2} y\right|_{T_{x}^{2}}^{2}\right)+2 b\left|D^{2} y\right|^{2}+\left\langle D^{2} y, L(y)\right\rangle_{T_{x}^{2}}, \\
& \operatorname{tr} D^{2} y \operatorname{tr} D^{2}(H(y))=\frac{1}{2} H\left(\left(\operatorname{tr} D^{2} y\right)^{2}\right)+2 b\left(\operatorname{tr} D^{2} y\right)^{2}+\operatorname{tr} D^{2} y \operatorname{tr} L(y) .
\end{aligned}
$$

Combining the divergence formula with (3.29) and (3.30), we obtain (3.23).

LEMMA 3.3. Let $y \in H^{4}(\Omega)$ satisfy the following conditions:

$$
\left\{\begin{array}{l}
y \in H_{\Gamma_{0}}^{2}(\Omega), \quad \Delta^{2} y \in L^{2}(\Omega), \\
\Delta y+(1-\mu) B_{1} y=v_{1} \in L^{2}\left(\Gamma_{1}\right), \\
\partial_{\nu} \Delta y+(1-\mu) B_{2} y=v_{2} \in L^{2}\left(\Gamma_{1}\right) .
\end{array}\right.
$$

Then we have

$$
\begin{aligned}
& -\int_{\Omega}\left[\Delta^{2} y-(1-\mu) \delta(k d y)\right] H(y) d x \\
& \leq-\frac{1}{2} \int_{\Omega} a(y, y) d x+C_{0} \int_{\Gamma_{1}}\left(\left|v_{1}\right|^{2}+\left|v_{2}\right|^{2}\right) d \Gamma \\
& \quad+\int_{\Omega} T\left(D^{2} y, L(y)\right) d x,
\end{aligned}
$$

where $C_{0}$ is a positive constant depending only on the domain $\Omega$.

Proof. For a simple reason, we start with $v_{1} \in H^{\frac{3}{2}}\left(\Gamma_{1}\right)$, and $v_{2} \in H^{\frac{1}{2}}\left(\Gamma_{1}\right)$. Since $y \in H^{4}(\Omega)$, by Lemma 3.1 and Lemma 3.2 , we have

$$
\begin{aligned}
\int_{\Omega} & {\left[\Delta^{2} y-(1-\mu) \delta(k d y)\right](H(y)) d x } \\
= & \int_{\Omega} b a(y, y) d x+\int_{\Gamma} v_{2}(H(y)) d \Gamma \\
& -\int_{\Gamma} v_{1} \partial_{\nu}(H(y)) d \Gamma+\frac{1}{2} \int_{\Gamma} a(y, y)\langle H, \nu\rangle d \Gamma+\int_{\Omega} T\left(D^{2} y, L(y)\right) d x
\end{aligned}
$$

Since $y=\partial_{\nu} y=0$ on $\Gamma_{0}$, by Yao [7, Lemma 2.1], we have

$$
\frac{\partial(H(y))}{\partial \nu}=\Delta y\langle H, \nu\rangle, \quad B_{1}=0, \quad \text { and } \quad H(y)=0 \quad \text { on } \Gamma_{0} \text {. }
$$


Thus,

$$
\begin{aligned}
\int_{\Omega}[ & \left.\Delta^{2} y-(1-\mu) \delta(k d y)\right](H(y)) d x \\
= & \int_{\Omega} a(y, y) d x+\int_{\Gamma_{1}} v_{2}(H(y)) d \Gamma \\
& -\int_{\Gamma_{1}} v_{1} \partial_{\nu}(H(y)) d \Gamma+\frac{1}{2} \int_{\Gamma} a(y, y)\langle H, \nu\rangle+\int_{\Omega} T\left(D^{2} y, L(y)\right) d x \\
\geq & \int_{\Omega} a(y, y) d x+\int_{\Gamma_{1}} v_{2}(H(y)) d \Gamma \\
& -\int_{\Gamma_{1}} v_{1} \partial_{\nu}(H(y)) d \Gamma+\frac{1}{2} \int_{\Gamma_{1}}(1-\mu)\left|D^{2} y\right|_{T_{x}^{2}}^{2}\langle H, \nu\rangle d \Gamma+\int_{\Omega} T\left(D^{2} y, L(y)\right) d x
\end{aligned}
$$

Now a straightforward computation shows that

$$
\begin{aligned}
\left|\partial_{\nu}(H(y))\right| & \leq|D H(D y, \nu)|+\left|D^{2} y(H, \nu)\right|, \\
\left|\partial_{\nu}(H(y))\right|^{2} & \leq 2\left(|D H|_{T_{x}}^{2}|D y|^{2}+\left|D^{2} y\right|_{T_{x}}^{2}|H|^{2}\right) .
\end{aligned}
$$

It follows that, for any $\lambda>0$,

$$
\begin{aligned}
\int_{\Gamma_{1}} v_{1} \partial_{\nu}(H(y)) d \Gamma \geq & -\lambda \int_{\Gamma_{1}}\left|v_{1}\right|^{2} d \Gamma-\frac{r^{2}}{2 \lambda} \int_{\Gamma_{1}}\left|\partial_{\nu} y\right|^{2} d \Gamma \\
& -\frac{R^{2}}{2 \lambda} \int_{\Gamma_{1}}\left|D^{2} y\right|_{T_{x}}^{2} d \Gamma
\end{aligned}
$$

where $r=\sup _{x \in \Gamma_{1}}|D H|_{T_{x}}$ and $R=\sup _{x \in \Gamma_{1}}|H|$, and

$$
\int_{\Gamma_{1}} v_{2}(H(y)) d \Gamma \geq-\lambda \int_{\Gamma_{1}}\left|v_{2}\right|^{2} d \Gamma-\frac{R}{4 \lambda} \int_{\Gamma_{1}}|D y|^{2} d \Gamma .
$$

Inserting (3.35), (3.36) into (3.34), we get

$$
\begin{aligned}
\int_{\Omega}[ & \left.\Delta^{2} y-(1-\mu) \delta(k d y)\right](H(y)) d x \\
\geq & \int_{\Omega} a(y, y) d x-\lambda \int_{\Gamma_{1}}\left(\left|v_{1}\right|^{2}+\left|v_{2}\right|^{2}\right) d \Gamma \\
& -\frac{1}{4 \lambda} \int_{\Gamma_{1}}\left(r^{2}\left|\partial_{\nu} y\right|^{2}+R^{2}|D y|^{2}\right) d \Gamma+\int_{\Omega} T\left(D^{2} y, L(y)\right) d x
\end{aligned}
$$

provided $\lambda \geq \frac{R^{2}}{\delta(1-\mu)}$. We obtain (3.32) by taking $\lambda>0$ large enough in (3.37) such that

$$
\int_{\Gamma_{1}}\left(r^{2}\left|\partial_{\nu} y\right|^{2}+R^{2}|D y|^{2}\right) d \Gamma \leq \lambda \int_{\Omega} a(y, y) d x \quad \forall y \in H_{\Gamma_{0}}^{2}(\Omega) .
$$

If $v_{1}, v_{2} \in L^{2}\left(\Gamma_{1}\right)$, then by a standard argument of density (see Lemma 3.1 in Rao [9]), the proof is complete.

Let $y$ be a smooth solution of the system (3.6)-(3.7). We define the associated energy by

$$
E(t)=\frac{1}{2}\left\{\int_{\Omega}\left[\left|y^{\prime}\right|^{2}+a(y, y)\right] d x+\int_{\Gamma}\left(\rho\left|y^{\prime}\right|^{2}+J\left|\partial_{\nu} y^{\prime}\right|^{2}\right) d \Gamma\right\}
$$


Then using equations (3.6), (3.7), and formulae (3.9), we have

$$
\frac{d}{d t} E(t)=-\left\|y^{\prime}\right\|_{H^{2}(\Gamma)}^{2}-\left\|\partial_{\nu} y^{\prime}\right\|_{H^{2}(\Gamma)}^{2} \leq 0
$$

Therefore, $E(t)$ is a nonincreasing function.

Theorem 3.1. Let Assumption B hold. For any smooth solution $y$ to problem (3.6) and (3.7), there exists a constant $K>0$ depending only on the initial data of $y$ such that the following rational energy decay rate holds:

$$
E(t) \leq E(0) \frac{2 K}{K+t} \quad \forall t>0 .
$$

Proof. Letting $0 \leq T<S<+\infty$, we multiply the plate equation in (3.6) from both sides by $E(t) H(y)$ and integrate over $\Omega \times[T, S]$ by parts. We then obtain on one hand

$$
\begin{aligned}
\int_{T}^{S} \int_{\Omega} E(t) H(y) y^{\prime \prime} d x d t \\
=-\left[\int_{\Omega} E(t) H(y) y^{\prime} d x\right]_{T}^{S}-\int_{T}^{S} \int_{\Omega} E^{\prime}(t) H(y) y^{\prime} d x d t \\
\quad+\frac{1}{2} \int_{T}^{S} \int_{\Omega} E(t) \operatorname{div}\left(H\left|y^{\prime}\right|^{2}\right) d x d t-\frac{1}{2} \int_{T}^{S} \int_{\Gamma_{1}} E(t)\left|y^{\prime}\right|^{2}\langle H, \nu\rangle d \Gamma d t
\end{aligned}
$$

By the Cauchy-Schwarz inequality we have

$$
\left|\int_{\Omega} H(y) y^{\prime} d x\right| \leq C_{1} E(t) .
$$

Then it follows that

$$
\begin{aligned}
& {\left[\int_{\Omega} E(t) H(y) y^{\prime} d x\right]_{T}^{S}-\int_{T}^{S} \int_{\Omega} E^{\prime}(t) H(y) y^{\prime} d x d t} \\
& \quad \geq-C_{1}\left(E^{2}(T)+E^{2}(S)\right)+C_{1} \int_{T}^{S} E^{\prime}(t) E(t) d t \\
& \quad \geq-2 C_{1} E^{2}(T)
\end{aligned}
$$

Inserting (3.41) into (3.39), we have

$$
\begin{aligned}
\int_{T}^{S} \int_{\Omega} E(t) H(y) y^{\prime \prime} d x d t \geq & -2 C_{1} E^{2}(T)+\bar{b} \int_{T}^{S} \int_{\Omega} E(t)\left|y^{\prime}\right|^{2} \\
& -\frac{R}{2} \int_{T}^{S} \int_{\Gamma_{1}} E(t)\left|y^{\prime}\right|^{2} d \Gamma d t
\end{aligned}
$$

where $\bar{b}=\min _{x \in \bar{\Omega}} b(x)$. 
On the other hand, by Lemma 3.3, we obtain

$$
\begin{aligned}
& -\int_{T}^{S} \int_{\Omega} E(t) H(y)\left[\Delta^{2} y+(1-\mu) \delta(k d y)\right] d x d t \\
& \leq-\frac{1}{2} \int_{T}^{S} \int_{\Omega} E(t) a(y, y) d x d t+\int_{T}^{S} E(t) \int_{\Omega} T\left(D^{2} y, L(y)\right) d x \\
& \quad+C_{0} \int_{T}^{S} \int_{\Gamma_{1}} E(t)\left(\left|\rho y^{\prime \prime}+y^{\prime}\right|^{2}+\left|J \partial_{\nu} y^{\prime \prime}+\partial \nu y^{\prime}\right|^{2}\right) d \Gamma d t
\end{aligned}
$$

Therefore, combining (3.42), (3.43) and the plate equation, we have

$$
\begin{aligned}
& \int_{T}^{S} E^{2}(t) d t \\
& \quad \leq 2 C_{1} E^{2}(T)+\int_{T}^{S} E(t) \int_{\Omega} T\left(D^{2} y, L(y)\right) d x \\
& \quad+C_{2} \int_{T}^{S} \int_{\Gamma_{1}} E(t)\left(\left|y^{\prime}\right|^{2}+\left|\partial_{\nu} y^{\prime}\right|^{2}+\left|y^{\prime \prime}\right|^{2}+\left|\partial_{\nu} y^{\prime \prime}\right|^{2}\right) d \Gamma d t
\end{aligned}
$$

We now eliminate the term $\int_{T}^{S} E(t) \int_{\Omega} T\left(D^{2} y, L(y)\right) d x$ in (3.44) by the assumption (3.22).

By the Cauchy inequality, we have

$$
\begin{aligned}
\left(\int_{\Omega} T\left(D^{2} y, L(y)\right) d x\right)^{2} & \leq \int_{\Omega} T\left(D^{2} y, D^{2} y\right) d x \int_{\Omega} T(L(y), L(y)) d x \\
& =\int_{\Omega} a(y, y) d x \int_{\Omega} T(L(y), L(y)) d x
\end{aligned}
$$

On the other hand, it is not difficult to obtain

$$
\begin{aligned}
& T(R(D y, \cdot H, \cdot), R(D y, \cdot H, \cdot)) \\
& \quad=(1-\mu)|H|^{2} k^{2}|D y|^{2}+\mu(H(y))^{2} k^{2} \leq k^{2}|H|^{2}|D y|^{2}
\end{aligned}
$$

and

$$
T\left(l_{D y} D^{2} H, l_{D y} D^{2} H\right) \leq\left|D^{2} H\right|^{2}|D y|^{2} .
$$

From (3.46), (3.47), by the Cauchy inequality again, we have

$$
\begin{aligned}
\int_{\Omega} T(L(y), L(y)) d x & \leq\left(\theta_{2}+\theta_{3}\right)^{2} \int_{\Omega}|D y|^{2} d x \\
& \leq \theta_{1}^{2}\left(\theta_{2}+\theta_{3}\right)^{2} \int_{\Omega} a(y, y) d x \\
& \leq 2 \theta_{1}^{2}\left(\theta_{2}+\theta_{3}\right)^{2} E(t) .
\end{aligned}
$$

Combining inequalities (3.45) and (3.48) yields

$$
\int_{T}^{S} \int_{\Omega} T\left(D^{2} y, L(y)\right) d x d t \leq 2 \theta_{1}\left(\theta_{2}+\theta_{3}\right) \int_{T}^{S} E^{2}(t) d t .
$$


By (3.44), (3.49) and (3.22), we get

$$
\begin{aligned}
& \int_{T}^{S} E^{2}(t) d t \leq 2 C_{1} E^{2}(T) \\
& \quad+C_{2} E(T) \int_{T}^{S} \int_{\Gamma_{1}}\left(\left|y^{\prime}\right|^{2}+\left|\partial_{\nu} y^{\prime}\right|^{2}+\left|y^{\prime \prime}\right|^{2}+\left|\partial_{\nu} y^{\prime \prime}\right|^{2}\right) d \Gamma d t
\end{aligned}
$$

where the constants $C_{1}, C_{2}$ may be different from those in (3.44).

Now define the energy of high-order $E_{1}(t)$ by

$$
E_{1}(t)=\frac{1}{2}\left\{\int_{\Omega}\left(a(y, y)+\left|y^{\prime \prime}\right|^{2}\right)+J \int_{\Gamma_{1}}\left|\partial_{\nu} y^{\prime \prime}\right|^{2} d \Gamma+\rho \int_{\Gamma_{1}}\left|y^{\prime \prime}\right|^{2} d \Gamma\right\}
$$

Then by Lemma $3.1, \frac{d E_{1}(t)}{d t} \leq 0$. Thus,

$$
\int_{T}^{S} E^{2}(t) d t \leq K E(t) E(0)
$$

where we have put $K=2 C_{1}+C_{2}+C_{2}\left\|E_{1}(0)\right\|^{2} /\left\|u_{0}\right\|^{2}$.

Finally, we deduce the rational energy decay rate from (3.51) according to the following classical result (see Komornik [10] and Lagnese [11]).

LEMma 3.4. Let $E: R^{+} \rightarrow R^{+}$be a nonincreasing function. Assume that there exists a positive constant $K$ such that

$$
\int_{T}^{\infty} E^{2}(t) d t \leq K E(0) E(T), \quad \forall T>0 .
$$

Then we have

$$
E(t) \leq E(0) \frac{2 K}{K+t} \quad \forall t \geq 0
$$

4. Uniform stabilization of a simplified model. In this section, we consider the following simplified model, in which the bending moment of inertia of the boundary $J$ is neglected:

$$
\begin{cases}y^{\prime \prime}+\Delta^{2} y-(1-\mu) \delta(k d y)=0 & \text { in } \Omega \times[0, \infty), \\ y=\partial_{\nu} y=0 & \text { on } \Gamma_{0} \times[0, \infty), \\ \Delta y+(1-\mu) B_{1} y=-\partial_{\nu} y^{\prime} & \text { on } \Gamma_{1} \times[0, \infty), \\ \rho y^{\prime \prime}-\partial_{\nu} \Delta y-(1-\mu) B_{2} y=-y^{\prime} & \text { on } \Gamma_{1} \times[0, \infty)\end{cases}
$$

Let

$$
W=\left\{\begin{array}{l}
y \in H_{\Gamma_{0}}^{2}(\Omega), \quad \Delta^{2} y \in L^{2}(\Omega) \\
y^{\prime} \in H_{\Gamma_{0}}^{2}(\Omega),\left.\quad y^{\prime}\right|_{\Gamma_{1}} \in L^{2}\left(\Gamma_{1}\right) \\
\Delta y+(1-\mu) B_{1} y=v_{1} \in L^{2}\left(\Gamma_{1}\right), \\
\partial_{\nu} \Delta y+(1-\mu) B_{2} y=v_{2} \in L^{2}\left(\Gamma_{1}\right) .
\end{array}\right.
$$


REMARK 4.1. By the same arguments as in Sec. 3, we can get the well posedness and smoothness of the solution to the system (4.1), but we omit it.

Now let $y \in W \cap H^{4}(\Omega)$ be a solution of (4.1). Then we define the associated energy by

$$
E(t)=\frac{1}{2}\left\{\int_{\Omega}\left(\left|y^{\prime}\right|^{2}+a(y, y)\right) d x+\rho \int_{\Gamma_{1}}\left|y^{\prime}\right|^{2} d \Gamma\right\}
$$

Therefore,

$$
\frac{d}{d t} E(t)=-\int_{\Gamma_{1}}\left(\left|y^{\prime}\right|^{2}+\left|\partial_{\nu} y^{\prime}\right|^{2}\right) d \Gamma \leq 0,
$$

that is, $E(t)$ is nonincreasing and

$$
E(T)=E(0)-\int_{0}^{T} \int_{\Gamma_{1}}\left(\left|y^{\prime}\right|^{2}+\left|\partial_{\nu} y^{\prime}\right|^{2}\right) d \Gamma d t \quad \forall T>0 .
$$

For $T>0$, set

$$
\begin{aligned}
Q & =(0, T) \times \Omega ; \\
\Sigma_{0} & =(0, T) \times \Gamma_{0} ; \quad \Sigma=(0, T) \times \Gamma ; \\
\Sigma_{1} & =(0, T) \times \Gamma_{1} .
\end{aligned}
$$

LEMmA 4.1. Let $H$ satisfy (3.16). Let $y$ be a smooth solution to the problem (4.1) whose initial data $u_{0} \in W$. We then have the following identity:

$$
\begin{gathered}
\int_{Q} b\left(y^{\prime 2}+a(y, y)\right) d Q+\rho \int_{\Sigma_{1}} b y^{\prime 2} d \Sigma \\
=\rho \int_{\Sigma_{1}} b y^{\prime 2} d \Sigma+\frac{1}{2} \int_{\Sigma}\left[y^{\prime 2}-a(y, y)\right]\langle H, \nu\rangle d \Sigma \\
\quad+\int_{\Sigma}\left[\Delta y+(1-\mu) B_{1} y\right] \partial_{\nu}(H(y)) d \Sigma \\
-\int_{\Sigma}\left[\partial_{\nu}(\Delta y)+(1-\mu) B_{2} y\right] H(y) d \Sigma \\
-\left.\left(\int_{\Omega} H(y) y^{\prime} d x\right)\right|_{0} ^{T}+\operatorname{lot}(y),
\end{gathered}
$$

where $\operatorname{lot}(y)$ is the lower-order term with respect to the energy $E(t)$, defined by

$$
\operatorname{lot}(y)=\int_{Q} T\left(D^{2} y, L(y)\right) d Q
$$

Proof. By multiplying Eq. (4.1) by $H(y)$ and integrating over $\Omega \times[0, T]$, we obtain on one hand

$$
\begin{aligned}
\int_{Q} y^{\prime \prime} H(y) d Q= & \left(\int_{\Omega} y^{\prime} H(y) d x\right)_{0}^{T}+\int_{Q} b\left|y^{\prime}\right|^{2} d Q \\
& -\frac{1}{2} \int_{\Sigma_{1}}\langle H, \nu\rangle\left|y^{\prime}\right|^{2} d \Sigma
\end{aligned}
$$


On the other hand, by using Lemmas 3.1 and 3.2 , we have

$$
\begin{aligned}
\int_{Q}\left(\Delta^{2} y-\right. & (1-\mu) \delta(k d y)) H(y) d Q \\
= & \frac{1}{2} \int_{\Sigma} a(y, y)\langle H, \nu\rangle d \Sigma+\int_{Q} b a(y, y) d Q+\operatorname{lot}(y) \\
- & \int_{\Sigma}\left[\Delta y+(1-\mu) B_{1} y\right] \partial_{\nu}(H(y)) d \Sigma \\
& +\int_{\Sigma}\left[\partial_{\nu}(\Delta y)+(1-\mu) B_{2} y\right] H(y) d \Sigma
\end{aligned}
$$

The combination of formulae (4.9), (4.10) with Eq. (4.1) yields identity (4.7).

We now have the following.

Theorem 4.1. Let Assumption A hold. For any solution $y$ to the system (4.1), there exist two positive constants $K$ and $\omega$ such that

$$
E(t) \leq K E(0) e^{-\omega t}, \quad \forall t>0
$$

Proof. To get the uniform stabilization, it will suffice to prove that there are a time $T>0$ and a constant $C_{T}$, which is independent of the solution $y$, such that

$$
E(T) \leq C_{T} \int_{\Sigma_{1}}\left(\left|y^{\prime}\right|^{2}+\left|\partial_{\nu} y^{\prime}\right|^{2}\right) d \Sigma
$$

Indeed, if inequality (4.12) were true, then inequalities (4.4) and (4.12) together would imply

$$
E(T) \leq \frac{C_{T}}{1+C_{T}} E(0)
$$

so we have the uniform stabilization.

In the following, we prove inequality (4.12).

For $x \in \Gamma_{0}, y=\partial_{\nu} y=0$ imply $D y=0$. Then

$$
H(y)=0 \quad \forall x \in \Gamma_{0} .
$$

We therefore have

$$
D^{2} y(\tau, \tau)=D^{2} y(\tau, \nu)=0 \quad \forall x \in \Gamma_{0}
$$

It follows from (4.14) that

$$
\begin{gathered}
\partial_{\nu}(H(y))=D^{2} y(\nu, H)=\langle H, \nu\rangle D^{2} y(\nu, \nu)=\langle H, \nu\rangle \Delta y \quad \forall x \in \Gamma_{0}, \\
B_{1} y=B_{2} y=0 \quad \forall x \in \Gamma_{0}
\end{gathered}
$$


and

$$
\begin{aligned}
a(y, y) & =(1-u)\left(D^{2} y(\nu, \nu)\right)^{2}+\mu\left(D^{2} y(\nu, \nu)\right)^{2} \\
& =\left(D^{2} y(\nu, \nu)\right)^{2}=(\Delta y)^{2} \quad \forall x \in \Gamma_{0} .
\end{aligned}
$$

By (4.13)-(4.17), we obtain

$$
\begin{gathered}
\frac{1}{2} \int_{\Sigma_{0}}\left[y^{\prime 2}-a(y, y)\right] d \Sigma+\int_{\Sigma_{0}}\left[\Delta y+(1-\mu) B_{1} y\right] \partial_{\nu}(H(y)) d \Sigma \\
\quad-\int_{\Sigma_{0}}\left[\partial_{\nu}(\Delta y)+(1-\mu) B_{2} y\right] H(y) d \Sigma \\
=-\frac{1}{2} \int_{\Sigma_{0}}(\Delta y)^{2} d \Sigma+\int_{\Sigma_{0}}(\Delta y)^{2}\langle H, \nu\rangle d \Sigma \leq 0
\end{gathered}
$$

since $\langle H, \nu\rangle \leq 0$ for $x \in \Gamma_{0}$.

Since $\left|\partial_{\nu}(H(y))\right| \leq C\left(|D y|+\left|D^{2} y\right|\right)$ for $x \in \Gamma_{1}$, we have for any $\varepsilon>0$

$$
\begin{aligned}
& \left|\int_{\Sigma_{1}} \partial_{\nu} y^{\prime} \partial_{\nu}(H(y)) d \Sigma\right| \\
& \quad \leq \varepsilon \int_{\Sigma_{1}}\left|D^{2} y\right|^{2} d \Sigma+C_{\varepsilon} \int_{\Sigma_{1}}\left|\partial_{\nu} y^{\prime}\right|^{2} d \Sigma+\int_{\Sigma_{1}}|D y|^{2} d \Sigma \\
& \quad \leq \varepsilon \int_{\Sigma_{1}}\left|D^{2} y\right|^{2} d \Sigma+C_{\varepsilon} \int_{\Sigma_{1}}\left|\partial_{\nu} y^{\prime}\right|^{2} d \Sigma+C \int_{0}^{T}\|y\|_{H^{3 / 2}(\Omega)}^{2} d t \\
& \quad=\varepsilon \int_{\Sigma_{1}}\left|D^{2} y\right|^{2} d \Sigma+C_{\varepsilon} \int_{\Sigma_{1}}\left|\partial_{\nu} y^{\prime}\right|^{2} d \Sigma+\operatorname{lot}(y) .
\end{aligned}
$$

In addition, it is easy to check that

$$
\begin{aligned}
& -\int_{\Sigma_{1}}\left(\rho y^{\prime \prime}+y^{\prime}\right) H(y) d \Sigma \\
& \quad=-\rho\left(\int_{\Gamma_{1}} y^{\prime} H(y) d \Gamma\right)^{T}+\rho \int_{\Sigma_{1}} y^{\prime} H\left(y^{\prime}\right) d \Sigma-\int_{\Sigma_{1}} y^{\prime} H(y) d \Sigma \\
& \quad \leq C E(0)+C \int_{\Sigma_{1}}\left(\left|y^{\prime}\right|^{2}+\left|\partial_{\nu} y^{\prime}\right|^{2}\right) d \Sigma+\operatorname{lot}(y) .
\end{aligned}
$$

Since $\Gamma_{1}$ is closed, there is $\eta>0$ such that

$$
\langle H, \nu\rangle \geq \eta \quad \forall x \in \Gamma_{1} .
$$


Inserting inequalities (4.18)-(4.20) into identity (4.7), we obtain via the boundary conditions in (4.1) and inequality (4.21)

$$
\begin{aligned}
\int_{Q} b\left(y^{\prime 2}\right. & +a(y, y)) d Q+\rho \int_{\Sigma_{1}} b y^{\prime 2} d \Sigma \\
\leq & C \int_{\Sigma_{1}}\left|y^{\prime}\right|^{2} d \Sigma-\frac{(1-\mu) \eta}{2} \int_{\Sigma_{1}}\left|D^{2} y\right|^{2} d \Sigma \\
& -\int_{\Sigma_{1}} \partial_{\nu} y^{\prime} \partial_{\nu}(H(y)) d \Sigma-\int_{\Sigma_{1}}\left(\rho y^{\prime \prime}+y^{\prime}\right) H(y) d \Sigma \\
& -\left(\int_{\Omega} y^{\prime} H(y) d x\right)_{0}^{T}+\operatorname{lot}(y) \\
\leq & \left(\varepsilon-\frac{(1-\mu) \eta}{2}\right) \int_{\Sigma_{1}}\left|D^{2} y\right|^{2} d \Sigma \\
& +C E(0)+C_{\varepsilon} \int_{\Sigma_{1}}\left(\left|y^{\prime}\right|^{2}+\left|\partial_{\nu} y^{\prime}\right|^{2}\right) d \Sigma+\operatorname{lot}(y) .
\end{aligned}
$$

By setting $0<\varepsilon \leq(1-\mu) \eta / 2$, it follows from (4.22), (4.4), and (3.17) that

$$
b_{0} T E(T) \leq b_{0} \int_{0}^{T} E(t) d t \leq C E(T)+C_{\varepsilon} \int_{\Sigma_{1}}\left(\left|y^{\prime}\right|^{2}+\left|\partial_{\nu} y^{\prime}\right|^{2}\right) d \Sigma+\operatorname{lot}(y),
$$

that is,

$$
E(T) \leq \frac{C_{\varepsilon}}{b_{0} T-C} \int_{\Sigma_{1}}\left(\left|y^{\prime}\right|^{2}+\left|\partial_{\nu} y^{\prime}\right|^{2}\right) d \Sigma+\operatorname{lot}(y)
$$

where $T>0$ is appropriately large. By (4.4) again,

$$
E(0) \leq C_{T} \int_{\Sigma_{1}}\left(\left|y^{\prime}\right|^{2}+\left|\partial_{\nu} y^{\prime}\right|^{2}\right) d \Sigma+\operatorname{lot}(y)
$$

Finally, we eliminate the lower-order term in (4.24) by the classical method, compactness-uniqueness.

Let $T>0$ be large but fixed. If this were not the case, there would exist a sequence of solutions $\left\{y_{n}\right\}$ such that

$$
E_{n}(0)=1 \text { and } \int_{\Sigma_{1}}\left(\left|y_{n}^{\prime}\right|^{2}+\left|\partial_{\nu} y_{n}^{\prime}\right|^{2}\right) d \Sigma \rightarrow 0
$$

Since $\operatorname{lot}\left(y_{n}\right)$ are lower order, we may assume that

$$
\begin{gathered}
y_{n} \rightarrow y_{0} \quad \text { in } H^{1}\left(0, T ; H^{2}(\Omega)\right) ; \\
\lim _{n \rightarrow \infty} \operatorname{lot}\left(y_{n}\right)=y_{0} .
\end{gathered}
$$

It follows from (4.24), (4.25), and (4.27) that

$$
\begin{aligned}
E(0)\left(y_{n}-y_{m}\right) \leq & C_{T} \int_{\Sigma_{1}}\left(\left|y_{n}^{\prime}-y_{m}^{\prime}\right|^{2}+\left|\partial_{\nu}\left(y_{n}^{\prime}-y_{m}^{\prime}\right)\right|^{2}\right) d \Sigma \\
& +\operatorname{lot}\left(y_{n}-y_{m}\right) \rightarrow 0 \quad(\text { as } n, m \rightarrow \infty)
\end{aligned}
$$

This means that

$$
y_{n} \rightarrow y_{0} \quad \text { in } H^{1}\left(0, T ; H^{2}(\Omega)\right)
$$


We then get a solution $y_{0}^{\prime}$ to (4.1) that satisfies

$$
y_{0}^{\prime}=\partial_{\nu} y_{0}^{\prime}=0 \quad \forall x \in \Gamma_{1} .
$$

It is not hard to check from the boundary conditions in (4.1) that $y_{0}^{\prime}$ is a solution to the problem

$$
\begin{cases}u^{\prime \prime}+\Delta^{2} u-(1-\mu) \delta(k d u)=0 & \text { in }(0, T) \times \Omega, \\ u=\partial_{\nu} u=0 & \text { on }(0, T) \times \Gamma_{0}^{\prime}, \\ u=D u=D^{2} u=D^{3} u=0 & \text { on }(0, T) \times \Sigma_{1} .\end{cases}
$$

By the exact controllability in Yao [7], $y_{0}=0$. However, relations (4.24), (4.25), and (4.27) imply that $\operatorname{lot}\left(y_{0}\right)=1$. This conflict shows that there is $C_{T}>0$, independent of the solution $y$, such that

$$
E(0) \leq C_{T} \int_{\Sigma_{1}}\left(\left|y^{\prime}\right|^{2}+\left|\partial_{\nu} y^{\prime}\right|^{2}\right) d \Sigma .
$$

Therefore, inequality (4.12) is true.

\section{REFERENCES}

[1] B. Rao, Stabilization of elastic plate with dynamical boundary control, SIAM J. Control Optim. 36, 148-163 (1998)

[2] P. F. Yao, On the observability inequality for exact controllability of wave equations with variable coefficients, SIAM J. Control Optim. 37, 1568-1599 (1999)

[3] E. Hebey, Sobolev Spaces on Riemannian Manifolds, Lecture Notes in Mathematics, 1635, SpringerVerlag, Berlin, Heidelberg, 1996

[4] M. E. Taylor, Partial Differential Equations I, Springer-Verlag, New York, 1996

[5] H. Wu, C. L. Shen, and Y. L. Yu, An Introduction to Riemannian Geometry (in Chinese), Univ. of Beijing, 1989

[6] P. F. Yao, On the shallow shell equations, to appear

[7] P. F. Yao, Observability inequalities for the Euler-Bernoulli plate with variable coefficients, Contemporary Mathematics 268, 383-406 (2000)

[8] P. F. Yao, Observability inequalities for shallow shells, SIAM J. Control Optim. 38, 1729-1756 (2000)

[9] B. Rao, Stabilization of Kirchhoff plate equation in star-shaped domain by nonlinear boundary feedback, Nonlinear Anal. 20, 605-626 (1993)

[10] V. Komornik, Exact controllability and stabilization, The multiplier method, Masson, Paris, 1994

[11] J. E. Lagnese, Boundary stabilization of thin plates, SIAM, Philadelphia, PA, 1989

[12] A. Pazy, Semigroups of linear operators and applications to partial differential equations, SpringerVerlag, New York, 1983 